NP, IT, Proceedings, International Conference on Radiation-Tolerant Scintillators and Detectors, Tallahassee, FL; April 28 - May 2, 1992; Rad. Phys. Chem.

\title{
EARLY EVENTS FOLLOWING RADIOLYTIC AND PHOTOGENERATION OF RADICAL CATIONS IN HYDROCARBONS ${ }^{\dagger}$
}

\author{
D. W. WERST* and A. D. TRIFUNAC
}

Chemistry Division, Argonne National Laboratory, Argonne, IL 60439

ANL/CHM/CP -75982

DE93 003010

Short running title:

\section{RADIOLYTIC AND PHOTOGENERATION OF RADICAL CATIONS}

Communications should be sent to:

Dr. David W. Werst

Chemistry Division

Argonne National Laboratory

9700 S. Cass Avenue

Argonne, IL 60439

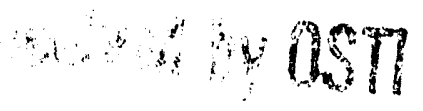

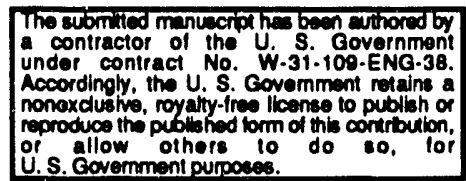

†Work performed under the auspices of the Office of Basic Energy Sciences, Division of Chemical Science, US-DOE under contract number W-31-109-ENG-38. 


\title{
EARLY EVENTS FOLLOWING RADIOLYTIC AND PHOTOGENERATION OF RADICAL CATIONS IN HYDROCARBONS $\dagger$
}

\author{
D. W. WERST and A. D. TRIFUNAC \\ Chemistry Division, Argonne National Laboratory, Argonne, IL 60439
}

\begin{abstract}
Real-time studies in hydrocarbons have revealed a richness of chemistry involving the initial ionic species produced in radiolysis and photoionization. A modified radical cation mechanism patterned after the core mechanism for alkane radiolysis - formation of radical cations and their disappearance via ion-molecule reactions - is capable of explaining a wide range of observations in high-energy photochemistry, and thus unifies two high-energy regimes. Fundamental studies of radical cations suggest strategies for mitigating radiation effects in materials.
\end{abstract}

\section{INTRODUCTION}

In this perspective we outline the fundamental early events in hydrocarbon radiolysis and photoionization with a view towards illuminating the microscopic origins of radiation damage in materials and possible ways of mitigating such damage. Our vantage point in the Radiation and Photochemistry Group at Argonne derives from real-time experimental studies of highenergy chemistry involving radical cations. Radical cations are the primary species in radiationinduced chemistry, and their fate determines the impact, major or minor, of the initial ionic events on radiation processes in materials. In this account we also explore instances of correspondence between alkane radiolysis and photoionization of aromatic hydrocarbons in an attempt to simplify and provide a more unified framework for the mechanistic interpretation of photolytic and radiolytic results.

Figure 1 is a coarse sketch of the fundamental processes following an ionization event in a hydrocarbon medium (e.g., hexane). Chemically, the most consequential initial event is charge separation - formation of radical cation, electron pairs. The positive and negative charges separate, mostly due to the motion of the highly mobile electron which has considerable excess kinetic energy immediately following ionization. The radical cation may also be formed with excess electronic/vibrational energy that can open up reaction channels not available to relaxed cations. All of the excess energy is disposed of via electron thermalization, unimolecular dissociation, vibrational relaxatinn and other energy-degradation steps within the first picosecond.

On the timescale accessible to real-time experiments, one is dealing with thermalized ion pairs and witnessing the end of nonhomogeneous processes such as geminate electron recombination - complete within several picoseconds in room-temperature nonpolar liquids (Warman, 1981). More than $95 \%$ of the cations and electrons undergo geminate recombination in alkanes because the low-dielectric liquids provide little shielding of the Coulombic attraction between the electron and its positive partner. The few percent of ions (free ions) which escape their geminate partners undergo homogeneous recombination on a slower timescale. 


\section{Event}

Ionization, Excitation

Charge separation, e- thermalization

Cation fragmentation

Energy transfer, Energy relaxation

Geminate e- recombination

e- scavenging

Positive ion scavenging

Geminate cation, anion recombination

lon-molecule reactions

Free ion (homogeneous) recombination

Spin relaxation

Neutral radical reactions

Products

Fig. 1.

The disappearance of the initial ionic species is remote from the eventual endpoint - the distribution of stable radiolysis products. The latter is the result of interest, however, in many applications, for it characterizes the actual chemical and physical modifications to the material. Product studies have been carried out in pure alkanes and a very few mixed systems, and they show the major products to be derived from radical coupling and disproportionation reactions (Foldiak, 1981) - i.e., mainly the result of second-order neutral radical reactions occurring on the microsecond timescale and later.

Successful strategies for mitigating radiation effects, nevertheless, must encompass all of the events depicted in Figure 1. Indeed, the best hope for affecting the final outcome lies in the ability to control events as early in time as possible, to influence the origins of the high-energy chemistry. Prediction of events, especially in complex mixed systems, requires understanding of the fate and chemical transformations of the initial species, which are ionic.

Referring to Figure 1, most of the ion chemistry in pure alkane liquids is terminated very early in time by geminate electron recombination - on a timescale barely accessible to experiment (with current technologies) and allowing little time for chemical transformations of thermalized positive ions. However, in real systems impurities or additives can scavenge electrons and slow down the kinetics considerably. In solids charged species can be trapped for long periods of time. Experimentally, ion scavengers and solid matrices are often employed for some of the same reasons: to understand mixed systems, to slow down the kinetics, to distinguish positive 
ions from negative ions, to isolate reactive species and to act as scintillators. Below, we present a discussion of radiolysis and photoionization roughly organized around different experimental approaches, in particular those which utilize scintillator solutions.

\section{Pulse Radiolysis of Scintillator Solutions}

Our discussion is framed in terms of mixed systems, because that conforms to experimental advantage in most cases and is relevant to real systems, including scintillation plastics from which are fabricated ionization detectors used in high-energy physics. Scheme I translates Figure 1 into equations. RH is an alkane molecule. In radiolysis (fast electrons, $\gamma, \mathrm{X}$-rays, etc.) excitation is nondiscriminate, and the solvent absorbs all of the energy. Eqs $1-3$ dipict ionization, energy relaxation and geminate electron recombination.

\section{Scheme I}

$$
\begin{aligned}
& \mathrm{RH} \stackrel{\text { e- beam }}{\rightarrow} \mathrm{RH}^{\bullet+*}+\mathrm{e}^{-} \\
& \mathrm{RH}^{\bullet+*} \longrightarrow \mathrm{RH}^{\bullet+} \\
& \mathrm{RH}^{\circ+}+\mathrm{e}^{-} \rightarrow \mathrm{RH}^{*} \\
& \longrightarrow \mathrm{R}^{\bullet}, \mathrm{H}^{\bullet}, \mathrm{H}_{2} \text {, olefin } \\
& \mathrm{A}+\mathrm{e}^{-} \rightarrow \mathrm{A}^{--} \\
& \mathrm{D}+\mathrm{RH}^{\bullet+} \longrightarrow \mathrm{D}^{\bullet+}+\mathrm{RH} \\
& D^{*+}+e^{-} \rightarrow D^{*} \\
& \mathrm{RH}^{\bullet+}+\mathrm{A}^{\bullet-} \longrightarrow \mathrm{RH}+\mathrm{A}^{*} \\
& \mathrm{D}^{\bullet+}+\mathrm{A}^{\bullet-} \rightarrow \mathrm{D}\left(\mathrm{D}^{*}\right)+\mathrm{A}^{*}(\mathrm{~A}) \\
& \mathrm{D}^{*}\left(\mathrm{~A}^{*}\right) \rightarrow \mathrm{hv}+\mathrm{D}(\mathrm{A})
\end{aligned}
$$

Electron recombination is a source of alkane excited states, $\mathrm{RH}^{*}$. In cyclohexane, where the $\mathrm{S}_{1}$ excited state is a prominent species, we have determined that $90 \%$ of $\mathrm{RH}^{*}$ is formed via recombination and $\leq 10 \%$ via direct excitation (Sauer et al., 1988). Fragmentation of $\mathrm{RH}^{*}$ constitutes one, but not the sole source of alkyl radicals, eq 4 (Nafisi-Movaghar and Hatano, 1974; .Schwarz et al., 1981).

Fluorescence from $\mathrm{RH}^{*}$ is not a convenient experimental probe because the fluorescence quantum yields for alkane excited states are very low $(-0.1 \%)$ (Rothman et al., 1973), and formation of $\mathrm{RH}^{*}$ via electron recombination is unresolvably fast in most cases. However, measurements of the $\mathrm{RH}^{*}$ fluorescence yield (in the absence of scintillators) and fluorescence quenching by electron scavengers such as perfluorohydrocarbons were among the first tests that implicated fast transformation reactions of $\mathrm{RH}^{\circ+}$. That is, it was concluded that not all $\mathrm{RH}^{\circ+}$ react to give excited states (Jonah and Sauer, 1989; Sauer et al., 1990). Fast reactions of $\mathrm{RH}^{\circ+}$ dictate the course of the early ionic chemistry and are revealed in a variety of other experiments, along with other important details, which have allowed us to elaborate on the details of Scheme I.

Recombination-fluorescence Studies. Addition of an aromatic solute provides a valuable experimental handle for real-time studies, i.e., fluorescence. The aromatic solute, or scintillator, may act as electron acceptor and/or donor. Scheme I allows for the possibility that different acceptor and donor molecules, i.e., different negative-ion and positive-ion scavengers, are used. The obvious experimental dividend is that excited state formation can be followed via recombination reactions such as eq 8 , and the excited state, $A^{*}$, fluoresces with high quantum yield. In 
adddition, the recombination kinetics are much slower than for electron recombination, and thus experimental resolution of excited state formation is possible.

Recombination-fluorescence studies carried out in the picosecond to nanosecond domain allow us to probe solvent radical cation dynamics via excited state formation, eq 8 . Comparison of the excited state formation curves with calculations utilizing a stochastic Monte Carlo model have allowed test:ng of different distribution functions to describe the initial electron- $\mathrm{RH}^{\circ+}$ separation distance (S shmidt et al., 1989; Sauer et al., 1990). The same comparison confirms that fast reactions of $\mathrm{RH}^{\circ+}$ occur which frustrate excited state formation via ion recombination. The lifetime at room temperature of the thermalized solvent radical cation has been determined in this way in cyclohexane $\left(t_{1 / 2}=0.3 n s\right)$ and $n$-hexane $\left(t_{1 / 2}=3 n s\right)$.

The yields of scintillator excited states are smaller than predicted theoretically (Jonah et al., 1990; Sauer et al., 1990). This divergence can be partially explained by the involvement of triplets, i.e., cross recombination in regions containing multiple ion pairs in close proximity (multiple-ion-pair spurs). The additional inefficiency that is required may be explained by attributing part of the initial ion yield ( $\mathrm{G}$ (ions) $\approx 5$ per $100 \mathrm{eV}$ absorbed) to positive ions from the solvent (e.g., $\mathrm{c}-\mathrm{C}_{6} \mathrm{H}_{11}+$ in cyclohexane and $\mathrm{C}_{6} \mathrm{H}_{13}+$ in $\mathrm{n}$-hexane) that are unable to produce excited states.

Fluorescence-Detected Magnetic Resonance. As we have described, recombination-fluorescence experiments provide kinetic information and a measurement of excited state yields. Chemical insights depend on chemical intuition and information from other types of experiments. Chemical identification is usually the greatest challenge in time-resolved studies in the condensed phase, i.e., unequivocal structural characterization of very short-lived intermediates.

In a fluorescence-based technique developed by us, we have a powerful method for the structural characterization of transient radical ions in the condensed phase. This method is timeresolved fluorescence-detected magnetic resonance (FDMR) (Werst and Trifunac, 1991; Trifunac and Werst, 1991; Werst and Trifunac, 1992). Very briefly, FDMR takes advantage of the spin coherence of the geminate radical ion pairs which recombine to give the fluorescent excited state. Radiolysis creates ion pairs with singlet spin phasing, and in the FDMR experiment singlet ion pairs are converted to triplet ion pairs via the absorption of microwaves at resonant magnetic field. In other words, it is an electron spin resonance experiment, and the transitions are registered as the attenuation of the fluorescence intensity.

FDMR yields the superposed EPR spectra of the positive and negative ions that recombined to give the fluorescent state. The unique aspects of FMDR compared to conventional EPR is that optical detection gives it orders of magnitude greater sensitivity and time resolution on the nanosecond timescale is possible. Furthermore, FDMR is highly selective as it detects only geminate radical ions and is blind to other paramagnetic intermediates such as neutral radicals. One can obtain dynamic information with the caveat that the processes must occur within the geminate recombination time window for the reason that the fluorescence signal decays as recombination goes to completion.

FDMR experiments clearly show the formation of olefin radical cations in alkane radiolysis, specifically the olefin radical cation resulting from the loss of $\mathrm{H}_{2}$ from the parent alkane radical cation (Werst et al., 1986; Werst and Trifunac, 1987). Fragmentation of $\mathrm{RH}^{\circ+}$ to give the olefin radical cation is an excellent example of the need for the "chemical resolution" inherent in FDMR. Because the olefin radical cation has approximately the same diffusion rate as $\mathrm{RH}^{\circ+}$ and also recombines to give excited states, it is indistinguishable from the initial solvent radical cation in a recombination-fluorescence experiment (and in some types of cation-scavenging experiments as well). On the other hand, since $\mathrm{RH}^{\circ+}$ and the olefin radical cation have different hyperfine structure, they are readily distinguished by FDMR.

A different reaction channel that has a more significant effect on the initial solvent radical cation lifetime is the ion-molecule reaction between $\mathrm{RH}^{\circ+}$ and neutral alkane molecules, eq 11 . This reaction is assigned to proton transfer from $\mathrm{RH}^{\circ+}$ to $\mathrm{RH}$, which is 
$\mathrm{RH}^{\cdot+}+\mathrm{RH} \stackrel{\mathrm{H}^{+}}{\rightarrow} \mathrm{R}^{\cdot}+\mathrm{RH}_{2}^{+}$

supported most strongly by solid-state EPR studies of alkane radical cations (Toriyama et al., 1987; Stienlet and Ceulemans 1992). FDMR studies first showed unambiguously that the proton-transfer channel effectively determines the $\mathrm{RH}^{\bullet+}$ lifetime (barring electron-transfer scavenging reactions) (Trifunac et al., 1989; Werst et al., 1989). Proton transfer between carbon centers was expected to be slow (Kresge, 1975). More remarkably, FDMR studies showed that the rates of proton transfer vary by at least two orders of magnitude among simple alkanes. The FDMR results place an upper limit of 10-20 ns on the lifetime of $\mathrm{RH}^{\bullet+}$ in alkane liquids. Fast reactions of alkane radical cations, while slowed down, still occur in solids at cryogenic temperatures.

Thus it is apparent that the conversion of radical cations to carbonium ions via proton transfer, eq 11 , accounts for the inefficiency in excited state formation in recombination-fluorescence studies (vide supra). That is, $\mathrm{RH}_{2}{ }^{+}$does not recombine to give $\mathrm{A}^{*}$. The lifetimes of $0.3 \mathrm{~ns}$ and 3 ns determined by picosecond-fluorescence studies for the radical cations in cyclohexane and n-hexane, respectively, are in good qualitative agreement with the FDMR results.

Transient Absorption. Olefin radical cations have also been identified in electron-irradiated alkanes on the picosecond timescale by optical absorption (Le Motais and Jonah, 1989). This is a very challenging experiment because of overlap of broad, unstructured spectra of radicals, radical cations and excited states, which makes precise characterization of the complex early chemical processes in many alkanes by absorption impractical. In select cases where resolution is possible, the absorption results are consistent with FDMR and fluorescence results.

While picosecond absorption is a very general lechnique, it performs best for species with more structured absorption profiles. Excellent examples again occur in solutions of aromatic hydrocarbons. The prevalence of multiple-ion-pair spurs is evidenced by absorptions of aromatic triplet states at early times in scintillator solutions (Jonah and Sauer, unpubl.). Aromatic radical ions are easily identified via their absorption profiles (when the cation and anion bands are not coincident) and are being found to occur in some unexpected chemical sequences. For example, the radical anion of a commonly used electron scavenger, $\mathrm{CO}_{2}$, is found to react with perylene to give the perylene radical anion in hydrocarbon solvents (Sauer and Jonah, 1992). In $\mathrm{SF}_{6}$-saturated alcohol solvents, delayed formation of aromatic radical cations is observed, believed to be due to oxidation of aromatic solutes by $\mathrm{SF}_{5}{ }^{\circ}$, which is formed by dissociation of $\mathrm{SF}_{6}{ }^{\circ-}$ (Liu et al., 1992). These findings are significant because they constitute unexpected sources of delayed radical ion formation and reveal unanticipated consequences accompanying the use of certain electron scavengers.

Conductivity. A novel facet of hydrocarbon radiolysis best highlighted by conductivity studies is the occurrence in certain alkanes, such as cyclohexane and decalin, of an anomolous, highly mobile positive ion (Warman, 1981; Sauer et al., 1983; Sauer and Schmidt, 1987; Sauer et al., 1987). This species, which is too long-lived to be the radical cation, remains an enigma and still attracts the attention of radiation chemists. The high-mobility species is formed in radiolysis and also in photoionization experiments where the energy-absorbing species is an aromatic solute molecule. We have more to say about photoionization in the next section.

\section{Photoionization of Aromatic Solutes in Hydrocarbons}

We have trespassed onto somewhat divergent territory in order to give an idea of different experimental approaches and the kind of information that each can provide. Before proceeding to a summation of radical cation chemistry and radiation effects in materials, we shall briefly discuss photoionization of aromatic hydrocarbons.

The principal difference between radiolysis and photoionization is that the latter $\mathrm{i}$ ivolves resonant energy absorption by a solute. By analogy to Scheme I, we write Scheme II but omit any direct solvent participation. (AH is an aromatic hydrocarbon, such as anthracene, paraterphenyl, etc.) Another difference in photoionization is the ability to vary the energy by 
varying the frequency of the photons. Photoionization allows direct excitation of the aromatic species and gives the experimenter the ability to prepare the aromatic radical cation with a controlled amount of excess energy.

\section{Scheme II}

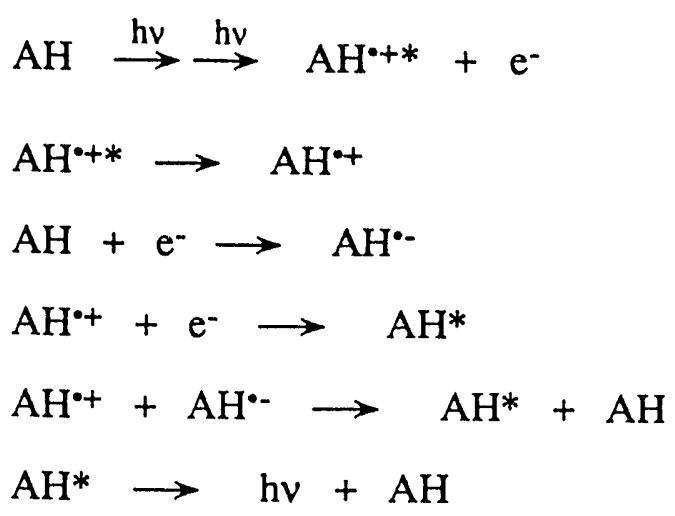

All of the experimental tools mentioned in section 2 have been used to study photoionization, including FDMR which gives insights into the photoionization step itself, eq 12 , as it is sensitive to the spin multiplicity of the intermediate excited states which is imprinted on the resulting ion pairs (Percy et al., 1989; Bakker and Trifunac, 1990).

In this section we shall focus on one specific question: Is it possible to unify many observations in radiolysis and high-energy photochemistry with a common ionic mechanism? That is, (1) does some correspondence exist between radiolysis and photoionization, and (2) how can we consolidate the proliferous mechanisms that at one time or another have enjoyed some vogue in photoionization studies?

This question led us to postulate a mechanism for photoionization by direct analogy to hydrocarbon radiolysis, which includes proton transfer from an aromatic radical cation to neutral solvent molecules, eq 18. As in radiolysis, there are competing channels available

$\mathrm{AH} \stackrel{\text { hv }}{\rightarrow} \stackrel{\text { hv }}{\rightarrow} \mathrm{AH}^{\cdot+*}+\mathrm{e}^{-}$

$\mathrm{AH}^{\bullet+*} \longrightarrow \mathrm{AH}^{\bullet+}$

$\mathrm{AH}^{\bullet+*}+\mathrm{RH} \rightarrow \mathrm{A}^{\cdot}+\mathrm{RH}_{2}^{+}$

to $\mathrm{AH}^{\bullet+}$, including recombination, but the ion-molecule reactions are needed to explain all of the observed products and intermediates. Unlike radiolysis, photoionization provides a direct means of examining the energetics of ionization for evidence of the involvement of unrelaxed radical cations, $\mathrm{AH}^{+* *}$.

Alternatively, results of photoexcitation with high-energy photons have been explained in terms of neutral excited states $\left(S_{n}, n>>1\right)$ which might undergo homolysis of a $C-H$ bond or interact with a neutral solvent molecule to give a solvent radical cation and solute radical anion. Each mechanism leads to specific predictions - concerning positive ion yields, electron yields, energy dependence and product yields. We have begun to test the relative merits of each mechanism on this basis, and the mounting evidence is strongly in favor of the radical cation mechanism embodied in eqs 12,13 and 18 (Loffredo et al., 1992). Therefore, especially with increasing energy, $\mathrm{AH}^{\circ}+$ shows a propensity to undergo chemical transformations that, inter alia, affect excited state yields.

It is instructive to briefly mention some of our product studies in photoionization since they also illuminate reasons for loss of scintillator during radiolysis of hydrocarbon, scintillator solutions. 
Product analysis following photoionization shows that consumption of aromatic solute molecules occurs because of reactions with solvent alkyl radicals, e.g., eq 19 (Loffredo and Trifunac, unpubl.). This loss mechanism must be minimized in order to

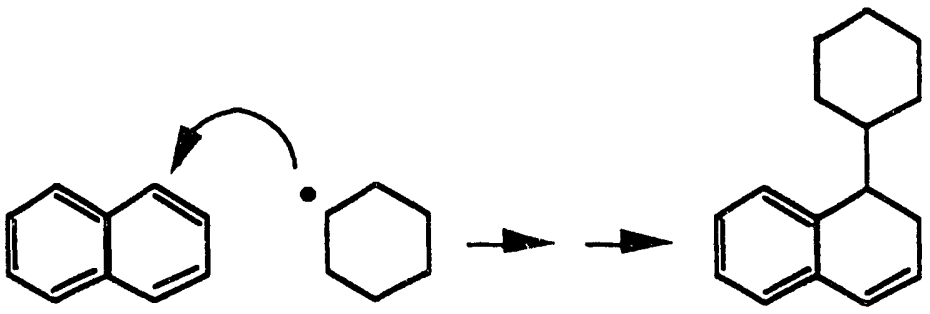

extend the lifetime of scintillator solutions - i.e., by reducing the production of alkyl radicals.

\section{CONCLUSIONS}

Ions play a pivotal role in determining the chemical transformations in materials exposed to high-energy radiation. Fundamental studies of transient species in real time have revealed the rich texture and features of the initial positive ion chemistry. Misunderstanding arises from considering structureless charge pairs with no chemical identity, because hydrocarbon radical cations and carbocations have different reactivities, result in different excited state yields and may exhibit entirely different modes of charge transport.

The most significant aspect of fundamental studies of radical cations in alkanes is that bimolecular transformation reactions of radical cations, i.e., ion-molecule reactions, are key. The implications for other fundamental studies in radiation chemistry are manifold. Two examples are: (1) In positive-ion-scavenging experiments, the chemical identity of the positive ion must be specified and whether reaction with the scavenger is electron transfer or proton transfer determined. (2) Any experimental probe of geminate ion recombination kinetics that depends on the integrity of the initial radical cation must take into account transformations of this cation.

The role of the initial radical cations in the net damage caused in materials exposed to radiation can be small or very great - depending in large part on the lifetime of the charge pairs in a given material. Energy partition between the electron and radical cation will determine the yield of "hot" cations, which could account for a significant part of the overall chemistry by reacting through channels not accessible to thermalized radical cations. Thermalized radical cations that do not promptly recombine have a propensity to undergo ion-molecule reactions, and thus the fate of radical cations in more complex systems will depend on the degree of isolation from other reactants and the nature of the matrix. This can mean spatial separation or something as subtle as molecular packing. Thus the radiation chemistry can vary considerably in different polymers, for example, depending on the electronic nature of the radical cations and the availability of nearby (nearest-neighbor) reactants.

Extrapolation from fundamental studies of prototype compounds to materials is an important step. Studies of alkanes are directly applicable to polyethylene-like polymers. Different prototypes can serve as model systems for other polymers. Clearly, fundamental studies in the polymers themselves, from the point of view of early ionic events, can and need to be carried out.

Unfortunately, no panacea will be found for avoiding harmful effects of radiation, because organic materials exposed to radiation give rise to inherently reactive species. The understanding gained from fundamental studies is an essential part of the important search for more radiationresistant materials and strategies. Real-time studies of transient species provide insights on how to slow down the inevitable degradation and manage the rate of damage.

More to the point, real materials are usually not single-component systems or will support modification by the addition of dopants. In multicomponent systems there are many more variables. Energy and charge will migrate to any existing lower energy traps, and, if these are more stable entities than the energy-absorbing species, then a gain has been achieved. We have 
many examples of this. For example, scavenging the highly reactive hexane radical cation with an olefin to form the olefin radical cation completely restores the full yield of excited states. In other words, one can find ways to degrade the chemical energy incrementally in ways that do not create unwanted material defects.

A wide range of approaches to making materials more radiation resistant can be summarized in a trivial way by generic recommendations, such as (1) promote recombination, (2) isolate reactants, (3) scavenge reactive species, etc. Future work will establish practical strategies for materials.

Acknowledgements. The authors acknowledge the members of the ANL Radiation and Photochemistry Group who carried out much of the research discussed in this account. We also thank the organizers of the RADDAM '92 conference for inviting radiation chemists to share in this dialog on radiation effects in scintillator materials. Work performed under the auspices of the Office of Basic Energy Sciences, Division of Chemical Science, US-DOE under contract number W-31-109-ENG-38.

\section{REFERENC.ES}

Bakker M. G. and Trifunac A. D. (1990) J. Phys. Chem., 95, 550.

Foldiak G., Ed. (1981) Radiation Chemistry of Hydrocarbons, Elsevier, Amsterdam.

Jonah C. D. and Sauer M. C., Jr. (1989) Radiat. Phys. Chem., 34, 497.

Jonah C. D., Sauer M. C., Jr. and Cooper R. (1990) J. Phys. Chem., 95, 728.

Kresge A. J. (1975) Acc. Chem. Res., $\underline{8}, 354$.

Le Motais B. C. and Jonah C. D. (1989) Radiat. Phys. Chem., 33, 505.

Liu A-D., Sauer M. C., Jr., Jonah C. D. and Trifunac A. D. (1992) J. Phys. Chem., in press.

Loffredo D. M., Liu A.-D. and Trifunac A. D. (1992) Radiat. Phys. Chem., in press.

Nafisi J. and Hatano Y. (1974) J. Phys. Chem., 78, 1899.

Percy L. T., Bakker M. G. and Trifunac A. D. (1989) J. Phys. Chem., 93, 4393.

Rothman W., Hirayama F. and Lipsky S. (1973) J. Chem. Phys., 58, 1300.

Sauer M. C., Jr., Trifunac A. D., McDonald D. B. and Cooper R. (1983) J. Phys. Chem., $\underline{88}$, 4096.

Sauer M. C., Jr. and Schmidt K. H. (1987) Radiat. Phys. Chem., 32, 281.

Sauer M. C., Jr., Schmidt K. H. and Liu A-D. (1987) J. Phys. Chem., 91, 4836.

Sauer M. C., Jr., Jonah C. D., Le Motais B. C. and Chernovitz A. C. (1988) J. Phys. Chem., $\underline{92,} 4099$.

Sauer M. C., Jr., Jonah C. D. and Naleway, C. A. (1990) J. Phys. Chem., 95, 730.

Sauer M. C., Jr. and Jonah C. D. (1992) J. Phys. Chem., in press.

Schmidt K. H., Sauer M. C., Jr., Lu Y. and Liu A-D. (1989) J. Phys. Chem., 94, 244.

Schwarz F. P., Smith D., Lias S. G. and Ausloos P. (1981) J. Chem. Phys., 75, 3800. 
Stienlet D. and Ceulemans J. (1992) J. Phys. Chem., in press.

Toriyama K, Nunome K. and Iwasaki M. (1987) J. Am. Chem. Soc., 109, 4496.

Trifunac A. D., Werst D. W. and Percy L. T. (1989) Radiat. Phys. Chem., 34, 547.

Trifunac A. D. and Werst D. W. (1991) Radical Ionic Systems, Lund A. and Shiotani M., Eds., Kluwer Academic Publishers, Netherlands, p. 195.

Warman J. M. (1981) The Study of Fast Processes an Transient Species by Electron Pulse Radiolysis, Baxendale J. H. and Busi F., Eds., Reidel, Boston, p. 433.

Werst D. W., Desrosiers M. F. and Trifunac A. D. (1986) Chem. Phys. Lett., 133, 201.

Werst D. W. and Trifunac A. D. (1987) J. Phys. Chem., 92, 1093.

Werst D. W., Bakker M. G. and Trifunac A. D. (1989) J. Am. Chem. Soc., 112, 40.

Werst D. W. and Trifunac A. D. (1991) J. Phys. Chem., 95, 3466.

Werst D. W. and Trifunac A. D. (1992) Electron Spin Resonance, Vol. 13A, Symons M. C. R., Ed., The Royal Society, Great Britain, p. 161.

\title{
DISCLAIMER
}

\begin{abstract}
This report was prepared as an account of work sponsored by an agency of the United States Government. Neither the United States Government nor any agency thereof, nor any of their employees, inakes any warranty, express or implied, or assumes any legal liability or responsibility for the accuracy, completeness, or usefulness of any information, spparatus, product, or process disclosed, or represents that its use would not infringe privately owned rights. Reference herein to any specific commercial product, process, or service by trade name, trademark, manufacturer, or otherwise does not necessarily constitute or imply its endorsement, recommendation, or favoring by the United States Government or any agency thereof. The views and opinions of authors expressed herein do not necessarily state or reflect those of the United States Government or any agency thereof.
\end{abstract}



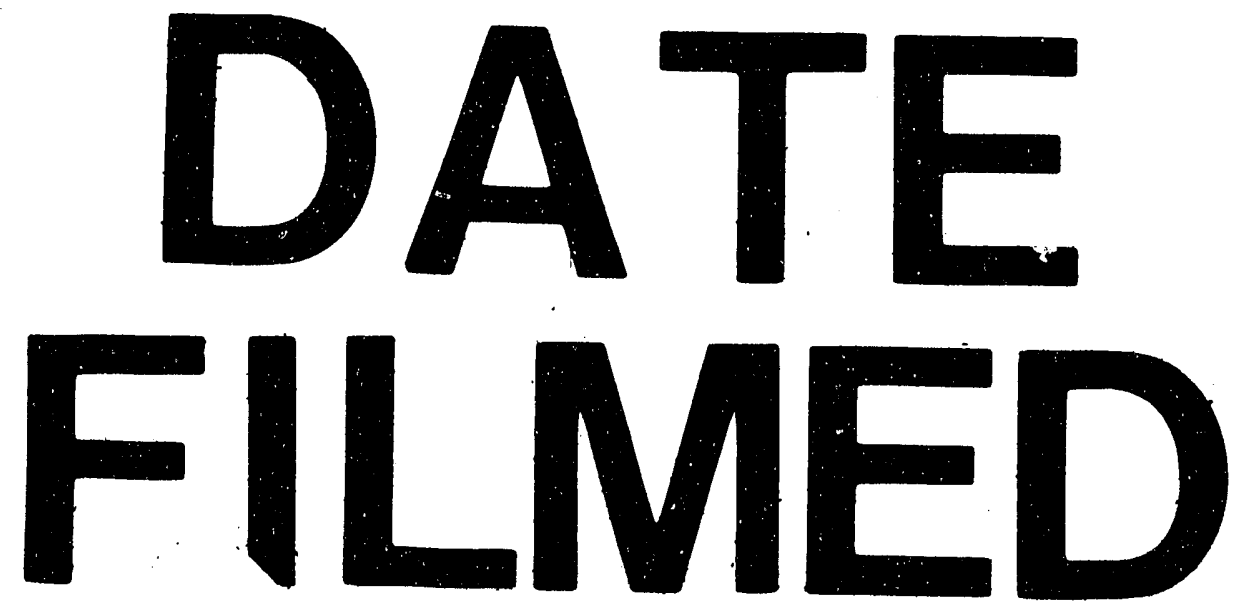

$02101 / 93$ 
